Professor Ivo Šteiner, MD, PhD, Editor

\title{
CASE 1-2013: FATAL MANIFESTATION OF MYELOPROLIFERATIVE DISEASE
}

\author{
Hana Ševčíková ${ }^{1}$, Radek Pelouch ${ }^{1}$, Adéla Matějková2, Petr Dulíček ${ }^{3}$ \\ Charles University in Prague, Faculty of Medicine and University Hospital Hradec Králové, Czech Republic: 1st Depart- \\ ment of Internal Medicine ${ }^{1}$, The Fingerland Department of Pathology ${ }^{2}$, 4th Department of Internal Medicine ${ }^{3}$
}

Key words: Myeloproliferation; Acute coronary syndrome

\section{Introduction}

Myeloproliferative disorders (MPDs) are defined as clonal stem cell diseases. The cardiovascular system is involved in 4 to $21 \%$ of MPD cases, presenting as acute ischemic coronary artery disease, valvular heart involvement, pericardial involvement, aortitis, thrombosis of major vessels, pulmonary embolism, systemic and pulmonary hypertension. Acute coronary syndrome may be the first manifestation of myeloproliferation.

\section{Clinical data}

A 33-year-old man was admitted to our intensive care unit with acute myocardial infarction. He had no history of arterial hypertension, hyperlipidemia or smoking. Several years earlier, he was assessed for asymptomatic thrombocytosis, and possible MPD was considered (bone marrow biopsy, positive mutation in the gene for JAK2); the "wait and watch" strategy (i.e. no therapy) was chosen. Physical examination on admission showed incipient cardiogenic shock. Urgent coronary angiography was indicated and left main stem occlusion was found. Thrombaspiration and bifurcation stenting LAD-RCx was done and intraaortic balloon contrapulsation inserted. During the coronary intervention the patient was intubated and arteficially ventilated. The platelet count on admission was $992 \times 10^{9 / 1}$ with gradual decrease during the hospital stay. A complex therapy of the cardiogenic shock continued - arteficial ventilation, intraaortic balloon contrapulsation, catecholaminergic support (including levosimendan) and continual hemoelimination. The Swan-Ganz catheter was inserted for optimalization of fluid therapy. Dual antiplatelet therapy, IIb/IIIa glycoprotein inhibitors and anticoagulation therapy were introduced. Mechanical circulation support was contraindicated for a febrile state. Despite complex therapy, the shock with multiple organ dysfunction syndrome progressed and the patient died after 11 days due to circulatory failure.

\section{Pathological findings}

Grossly, an extensive subacute transmural myocardial infarction dominated, involving most of the left ventricle. Apart from the infarction, the heart appeared basically normal (heart weight $410 \mathrm{~g}$ ). A metallic stent was implanted in the proximal left coronary artery. There were signs of heart failure - pulmonary congestion and edema, pericardial effusion $(100 \mathrm{ml})$, bilateral hydrothorax and congestion of the abdominal organs. There were multiple thrombi in the vascular system, affecting (among others) superior and inferior vena cava, both brachiocephalic veins and abdominal aorta, with multiple renal and splenic infarctions. However, practically no atherosclerosis was found. Microscopy confirmed subacute myocardial infarction affecting approximately $80 \%$ of the left ventricular myocardium. Multiple small intramyocardial vessels were occluded by fibrinous and thrombocytic thrombi. There were signs of a diffuse alveolar damage (ARDS) in the congested lungs. The bone marrow was hypercellular due to panhyperplasia of myeloid cells, particularly erythroid precursors and clusters of megakaryocytes. The latter were pleiomorphic, frequently with hyperlobulated nuclei. The bone marrow histology confirmed the diagnosis of myeloproliferative disorder - polycythemia vera like type.

\section{Discussion}

MPDs are neoplastic disorders characterized by proliferation of the myeloid stem cell. Polycythemia vera (PV, one of the MPD family) is characterized by erythrocytosis and thrombocytosis. PV usually affects old individuals, but $4-7 \%$ of patients are younger than 40 years (1). Other 


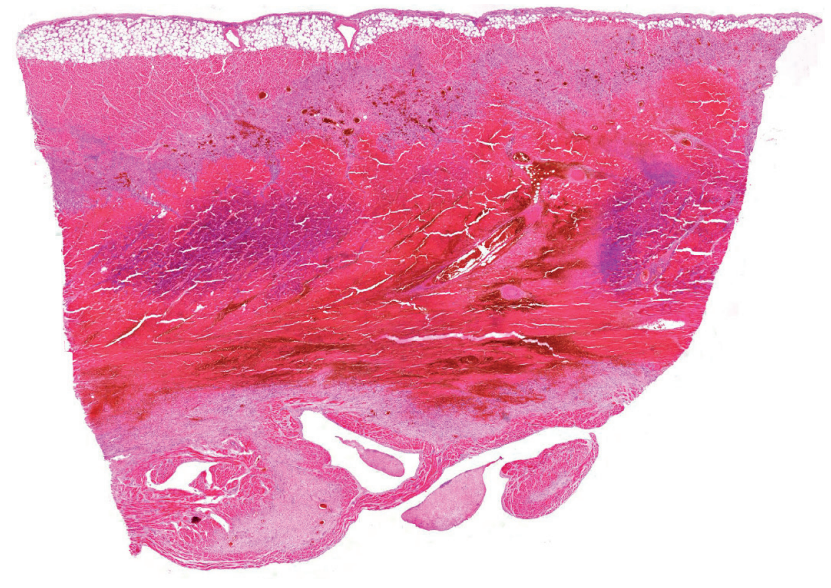

Fig. 1: Transmural myocardial infarction in the anterior wall of the left ventricle, modified by reperfusion (hematoxylin-eosin)

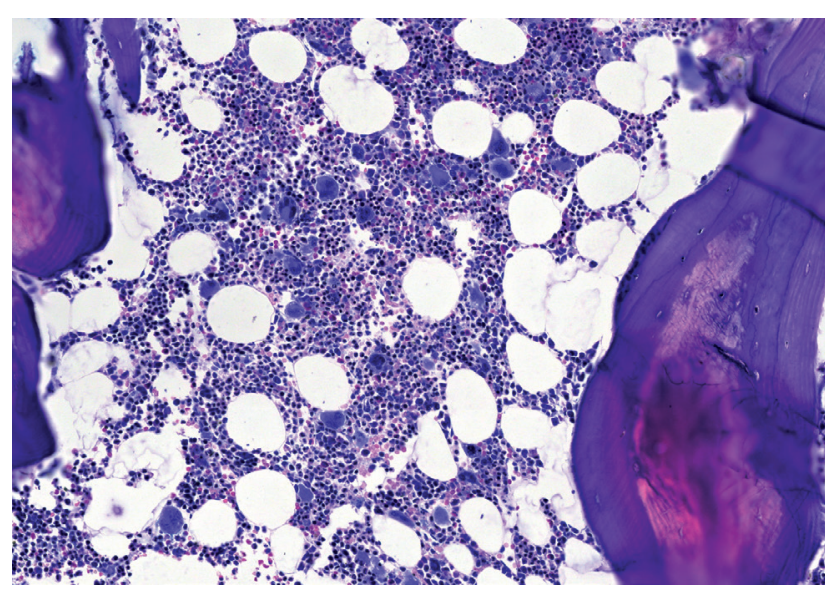

Fig. 2: Bone marrow biopsy (examined one year befor death) show the picture of incipient myeloproliferative syndrome of the polycythemia vera type (mild hypercellularity, panhyperplasia of myeloid cells, small noncohesive clusters of pleomorphic megakaryocytes without dysplastic changes). In addition, there were fine reticular fibers and minimal hemosiderin deposits (hematoxylin-eosin)

features of the disease include leukocytosis, splenomegaly, thrombotic or hemorrhagic complications, vasomotor disturbances, pruritus and risk of progression into acute leukemia (2). Bleeding and thrombosis (arterial or venous) are the primary causes of morbidity and mortality. Advanced age, prior history of thrombosis, hyperlipidemia, hypertension, smoking, diabetes mellitus and leukocytosis have been reported as risk factors for thrombosis. Leukocytosis is a consistent risk factor for coronary heart disease and in polycythemia vera it is associated with a higher risk of myocardial infarction (3). The cardiovascular system is involved in 4 to $21 \%$ of cases of myeloproliferative diseases. PV and essential thrombocytemia (ET) predispose to thrombotic complications in cerebral, digital, mesenteric, portal and retinal circulation. Coronary circulation may also be involved, with acute coronary syndrome or sudden death being the first manifestation of newly diagnosed or untreated PV. This, however, is very uncommon under the age of $40(4,5)$. The pathophysiologic mechanisms of trombus formation include an increased procoagulant activity of the platelets, changes in glycoproteins on the thrombocyte surface, or a possible deficiency of selective lipooxygenase (6). However the pathophysiology of thromboembolism is not fully understood (7). Thrombosis can occur without a severely abnormal platelet count (8). Treatment of patients with acute coronary syndromes and myeloproliferative disorders include reperfusion and antithrombotic therapy. Optimal pharmacologic and interventional management strategies remain unclear; the treatment can be difficult, particularly in the presence of bleeding. An elevated platelet count is an independent risk factor for poor outcome of the myocardial infarction. Given the results of the ECLAP study in PV, low-dose aspirin from the time of presentation in such a patient may possibly reduce the risk of myocardial infarction (5).

\section{Hematologist's comments}

The coagulation disorders in MPDs are rather complex and they can be the first manifestation of the disease as well as a later complication. The precise incidence of bleeding and thrombotic events is difficult to assess $(9,10)$. According to published data, thrombotic complications are more frequent (arterial, venous and microcirculation). In patients with PV and ET, thrombosis has been found in 12 to $39 \%$ and 11 to $25 \%$ respectively, at the time of diagnosis. Further on the incidence of thrombosis is ca. 1.2\%/patient/year in PV and 6.6\%/patient/year in ET. Bleeding at diagnosis has been reported with considerable variability: in 1.7 to $20 \%$ in PV and in 3.6 to $37 \%$ in ET. The incidence of bleeding thereof is close to zero in PV and $0.33 \%$ /patient/year in $\operatorname{ET}(11,12)$.

The pathogenesis is multifactorial; changes in all blood elements play a role in coagulopathy (13). Among them, an increased hematocrit, thrombocytemia, thrombocytopathies, and activation of leucocytes are the most important. The risk factors for thrombosis include age, previous thrombosis, inherited or acquired thrombophilia and common cardiovascular risk factors. The treatment is complex. Aspirin is recommended for primary prevention in patients with thrombocytosis and at least 1 risk factor. In the case of thrombosis, it is necessary to maintain the blood count within normal range, and anticoagulation is administered according to standard rules. Anticoagulation therapy is long-term, and often life-long. The classification of patients according to the risk of thrombosis is shown in Tab. 1 (12). 
Tab. 1: Stratification of patients according to thrombotic risk

\begin{tabular}{|l|c|c|c|c|c|}
\hline Magnitude of risk & Age & $\begin{array}{c}\text { Personal history of } \\
\text { thrombosis }\end{array}$ & $\begin{array}{c}\text { Additional risk } \\
\text { factor (RF) }\end{array}$ & $\begin{array}{c}\text { Cytoreductive } \\
\text { therapy }\end{array}$ & Antithrombotic \\
\hline High & $>60 \mathrm{y}$. & Positive & No & + & Aspirin, Warfarin \\
\hline Moderate & $<60 \mathrm{y}$. & Negative & Yes & According to RF & Aspirin \\
\hline Low & $<60 \mathrm{y}$. & Negative & No & - & Aspirin \\
\hline
\end{tabular}

\section{Message from the Editor (Prof. Šteiner)}

A young man with an untreated myeloproliferative disease - polycythemia vera - died from myocardial infarction. He had no classical risk factors of atherosclerosis (hyperlipidemia, hypertension, diabetes, smoking) and, indeed, the autopsy showed virtual absence of coronary atherosclerosis.

This is a rare case of coronary thrombosis with myocardial infarction as a lethal manifestation of polycythemia vera.

There are four major diagnostic entities recognized within the family of myeloproliferative disorders - chronic myelogenous leukemia, polycythemia vera, primary myelofibrosis, and essential thrombocythemia. Polycythemia vera usually affects patients over 50 years of age. The major anatomic changes stem from increased blood volume and viscosity brought about by the polycythemia (with low levels of serum erythropoietin). About $30 \%$ of patients with polycythemia vera develop thrombotic complications, usually affecting the brain or heart.

Roughly half of affected victims die from thrombosis or hemorrhage, and the other half from leukemia, myelofibrosis with anemia, congestive heart failure, or some other cause.

In our case, we can only speculate whether treatment of the known myeloproliferative disease would prevent the deadly complication.

\section{References}

1. Perea G, Remacha A, Besses C, et al. Is polycythemia vera a serious disease in young adults? Haematologica 2001; 86(5): 543-4

2. Tefferi A. Annual clinical updates in hematological malignancies: a continuing medical education series: polycythemia vera and essential thrombocythemia: 2011 update on diagnosis, risk stratification and management. Am J Hematol 2011; 86(3): 292-301.

3. De Stefano V, Za T, Rossi E, et al. Recurrent thrombosis in patients with polycythemia vera and essential thrombocythemia: incidence, risk factors, and effect of treatments. Haematol 2008; 93(3): 372-380.

4. Venegoni P, Cyprus G. Polycythemia and the heart. Texas Heart Institute Journal 1994; 21: 198-201.

5. Kelly SV, Burke RF, Lee KS, et al. Presentation of essential thrombocytosis in a 17-year-old man. Clinical Advances in Hematology \& Oncology 2008; 6(2): $133-9$.

6. Alioglu E, Tuzun N, Sahin F, et al. Non ST-segment elevation myocardial infarction in patient with essential thrombocythemia. Thrombosis journal 2009; 7: 1 .

7. Saif MW, Khan U, Greenberg BR. Cardiovascular manifestation of myeloproliferative disorders: A Review of the Literature. Hospital Physician 1999; July: 43-54.

8. Nanavati A, Patel N, Burke J. Thrombocytosis and coronary occlusion. J Am Coll Cardiol Intv. 2012; 5(6): 18-19.

9. Schafer AI. Bleeding and thrombosis in the myeloproliferative disorders. Blood 1984; 64: 1-12.

10. Landolfi R, Rocca B, Patron C. Bleeding and thrombosis in myeloproliferative disorders: mechanism and treatment. Critical reviews in Oncology-Hematology 1995; 20: 203-22.

11. Elliot MA, Tefferi A. Thrombosis and haemorrhage in polycythemia vera and Essentials thrombocythaemia. Br J Haematol 2004; 128: 275-290.

12. Cortelazzo S, Viero P, Finazzi G, et al. Incidence and risk factors for thrombotic complications in a historical cohort of 100 patients with essential thrombocythemia. Journal of Clinical Oncology 1990; 8: 556-562.

13. Landolfi R, Cipriani MC, Novarese L. Thrombosis and bleeding in polycythemia vera and essential thrombocythemia: pathogenic mechanism and prevention.Best Pract Res Clin Haematol 2006; 19(3): 617-633.

\section{Corresponding author:}

Hana Ševčíková, MD, 1st Department of Internal Medicine, University Hospital, Sokolská 581, 50005 Hradec Králové, Czech Republic; e-mail: hana.sevcikova@fnhk.cz 